\title{
Analysis of optically anisotropic properties of biological tissues under stretching based on differential Mueller matrix formalism
}

Hao-Wei Chen

Chih-Ling Huang

Yu-Lung Lo

You-Ren Chang 


\title{
Analysis of optically anisotropic properties of biological tissues under stretching based on differential Mueller matrix formalism
}

\author{
Hao-Wei Chen, ${ }^{a}$ Chih-Ling Huang, ${ }^{b}$ Yu-Lung Lo, ${ }^{a, c, *}$ and You-Ren Chang ${ }^{a}$ \\ ${ }^{a}$ National Cheng Kung University, Department of Mechanical Engineering, Tainan, Taiwan \\ ${ }^{\mathrm{b}}$ Kaohsiung Medical University, Center for Fundamental Science, Taiwan \\ 'National Cheng Kung University, Advanced Optoelectronic Technology Center, Tainan, Taiwan
}

\begin{abstract}
The optical properties of biological tissues under stretching are investigated using a full-field ellipsometry technique based on a differential Mueller matrix formalism. Traditional photoelastic-based formalism for extracting the linear birefringence (LB) properties of stretched anisotropic optical samples ignores the effects of the other optical properties of the sample. By contrast, in the formalism proposed in this study, the LB, linear dichroism (LD), circular birefringence (CB), circular dichroism (CD), and depolarization (Dep) properties are fully decoupled. Simulations are performed to evaluate the performance of the two formalisms in extracting the LB properties of optically anisotropic samples with different degrees of Dep, CB, LD, and CD. The practical feasibility of the proposed all-parameter decoupled formalism is then demonstrated using chicken breast muscle tissue. In general, the results show that both formalisms provide a reliable LB measurement performance for healthy chicken breast tissue under stretching. However, while the LB-only formalism has good robustness toward scattering, its measurement performance is seriously degraded for samples with high CB. Thus, of the two formalisms, the proposed all-parameter decoupled formalism provides a more effective approach for examining the anisotropic properties of biological tissues under stretching. () The Authors. Published by SPIE under a Creative Commons Attribution 3.0 Unported License. Distribution or reproduction of this work in whole or in part requires full attribution of the original publication, including its DOI. [DOI: 10.1117/1.JBO.22.3.035006]
\end{abstract}

Keywords: anisotropic; birefringence; Mueller matrix.

Paper 160661RR received Sep. 25, 2016; accepted for publication Feb. 22, 2017; published online Mar. 11, 2017.

\section{Introduction}

Biological tissues have unique and well-documented microscopic fibrous structures, which result in a pronounced birefringence property. ${ }^{1}$ The change in these structures under stretching results in a change in the birefringence. However, the birefringence of muscle tissue is related not only to the microscopic structure but also to the surrounding physical, biological, and chemical environment. ${ }^{2}$ As a result, changes in the muscle birefringence provide a useful indication of possible changes in the physical function, metabolism, and pathology of the tissue. Thus, the birefringence properties of biological tissue under stretching are of significant interest in a variety of disease diagnosis applications.

Cheong et $\mathrm{al}^{3}$ published a review paper on the optical properties of biological tissues and described the theoretical frameworks underlying the main experimental techniques used to analyze these properties in typical biological samples. Liao et al. ${ }^{4}$ used a CCD camera and Monte Carlo simulations to analyze the anisotropic optical properties of chicken heart tissue. They developed a rotating linear polarization imaging technique and obtained a set of new images of the parameters by fitting linear differential polarization (LDP) images pixel-by-pixel to an analytical expression. The observation results revealed that the heart muscles are aligned in different directions at different sections of the organ, and hence, the heart exhibits a strong

*Address all correspondence to: Yu-Lung Lo, E-mail: loyl@mail.ncku.edu.tw
LDP effect. Wood et al. ${ }^{5}$ presented a polarization birefringence technique for measuring the anisotropic characteristics of heart muscle fibers. The experimental results showed that the birefringence decreased in the infarcted region of the myocardium compared to the normal regions. Chen et al. ${ }^{6}$ used a polarizationsensitive optical coherence tomography (PS-OCT) technique to investigate the optical properties of chicken muscle tissue under stretching and showed that the birefringence increased with an increasing tensile force. Dobashi et al. ${ }^{7}$ examined the birefringence properties of anisotropic chitosan gels using a polarized light microscopy approach. The results showed that the difference in the birefringence intensity between the inner and outer parts of the chitosan gel was consistent with the change in orientation of the polymer aggregates in the corresponding domains. Csoka et al. ${ }^{8}$ fabricated human amyloid fibrils made of chitosan. The polysaccharide complexes and highly ordered long-chain carbohydrates of samples were observed by a polarization microscopy in order to clarify the complex proteinpolysaccharide structure of amyloid fibrils.

The studies above focus principally on the linear birefringence (LB) properties of biological tissue. However, in practice, biological tissues have not only LB properties but also circular birefringence $(\mathrm{CB})$, circular dichroism (CD), linear dichroism (LD), and scattering depolarization (Dep) effects. Due to the fact that $\mathrm{CB}$ and $\mathrm{CD}$ effects are quite rare in backscattering, ${ }^{9}$ the present study utilizes a full-field Mueller ellipsometry technique based on the differential Mueller matrix formalism proposed by the current group in Ref. 10 to conduct a more 
robust investigation of the anisotropic properties of biological tissue under stretching by transmission measurement system. In contrast to previous studies based on a simple LB-only model, ${ }^{11}$ the differential Mueller matrix formalism used in the present study decouples the effects of the CB, CD, LD, and Dep properties of the sample on the LB response, and therefore, enables the LB properties to be more reliably determined. The chicken breast muscle tissue is chosen for the stretching test because it has a well-defined texture direction, and the adipose tissue is also less than the other muscle tissue from porcine or bovine model. How the optical properties change while stretching the chicken breast muscle is studied. Simulations are performed to evaluate the performance of the traditional LB-only model and the proposed "all-parameter" decoupled model in extracting the LB properties of hypothetical anisotropic samples with various degrees of Dep, CB, LD, and CD. The practical feasibility of the proposed all-parameter decoupled model is then demonstrated by measuring the optical properties of chicken breast muscle tissue under tensile loads ranging from 0 to $1.0 \mathrm{~N}$.

\section{Materials and Methods}

\subsection{Mueller Matrix Polarimetry Method}

The traditional way to measure the stress and strain of materials by a photoelastic-based model is via observation of the LB property, and this method has been developed as a commercial instrument. If the material thickness and stress-optic coefficient of the material are known, the LB can be used to calculate the residual stress. ${ }^{12}$ However, in order to decouple more than LB property in a tested sample, a Mueller matrix polarimetry method is thus introduced.

Cameron et al. ${ }^{13}$ investigated the properties of turbid samples illuminated by a polarized light beam using a two-dimensional Mueller matrix method based on 49 images of the sample obtained under different combinations of the input and output analyzer polarization states. The experimental results obtained for a sample consisting of polystyrene beads suspended in deionized water were shown to be in good agreement with the numerical results. Consequently, Schaefer et al. ${ }^{14}$ proposed a more straightforward method for measuring the Mueller matrix parameters of the sample using the experimental setup shown in Fig. 1. In the proposed method, the sample is illuminated by six different input lights (four linear polarized incident lights with angles of $0,45,90$, and $135 \mathrm{deg}$, respectively, one right-hand polarized incident light, and one left-hand polarized incident light). For each input light, the intensity $I(\theta)$ of the optical beam incident on the detector is given by ${ }^{14}$

$I(\theta)=\frac{1}{2}(A+B \sin 2 \theta+C \cos 4 \theta+D \sin 4 \theta)$,

where $\theta$ is the angle of the rotating quarter waveplate in the analyzer (dashed-line box in Fig. 1) and $A, B, C$, and $D$ are constants given by $A=S_{0}+0.5 S_{1}, B=S_{3}, C=0.5 S_{1}$, and $D=0.5 S_{2}$, respectively, in which $S_{0}, S_{1}, S_{2}$, and $S_{3}$ are the Stokes parameters. As shown, Eq. (1) has the form of a truncated Fourier series consisting of a DC term, a second-order harmonic term, and two fourth-order harmonic terms. According to the Nyquist sampling theorem, a total of eight data points are required to determine the values of $A, B, C$, and $D$. For the configuration shown in Fig. 1, this is achieved by setting the quarter waveplate in the analyzer to rotation angles of $0,22.5,45,67.5$, $90,112.5,135$, and $157.5 \mathrm{deg}$, respectively. ${ }^{14}$ Having obtained the values of $A, B, C$, and $D$, the Stokes parameters can be determined algebraically as $S_{0}=A-C, S_{1}=2 C, S_{2}=2 D$, and $S_{3}=B$. Then, the 16 elements of the Mueller matrix for the sample of interest can be derived as

M

$=\frac{1}{2}\left[\begin{array}{llll}S_{1 \_0}+S_{2 \_} & S_{1 \_0}-S_{2 \_0} & S_{3 \_0}-S_{4 \_0} & S_{5 \_0}-S_{6 \_0} \\ S_{1 \_1}+S_{2 \_1} & S_{1 \_1}-S_{2 \_1} & S_{3 \_1}-S_{4 \_1} & S_{5 \_1}-S_{6 \_1} \\ S_{1 \_2}+S_{2 \_2} & S_{1 \_2}-S_{2 \_} & S_{3 \_2}-S_{4 \_2} & S_{5 \_2}-S_{6 \_} \\ S_{1 \_3}+S_{2 \_3} & S_{1 \_3}-S_{2 \_3} & S_{3 \_3}-S_{4 \_3} & S_{5 \_3}-S_{6 \_3}\end{array}\right]$,

where a $S_{i_{-} j}$ term is the $j$ 'th row value of the $i$ 'th output Stokes vector incoming from the sample.

\subsection{Anisotropic Property Extraction by Differential Mueller Matrix Model}

It was shown in Refs. 15 and 16 that the optical properties of depolarizing anisotropic media can be decoupled using a differential Mueller matrix derived through an eigenanalysis of the corresponding Mueller matrix. In calculating the differential matrix, a beam propagating along the $z$-axis in a right-handed Cartesian coordinate system is considered and the differential matrix (m) is related to the corresponding macroscopic Mueller matrix $(\mathrm{M})$ as follows:

$\mathrm{m}=(d \mathbf{M} / d z) \mathbf{M}^{-1}$,

where $\mathrm{M}$ is acquired via Mueller matrix polarimetry method. The eigenvalues of the macroscopic and differential matrices are derived as $\lambda_{\mathrm{M}}$ and $\lambda_{\mathrm{m}}$, respectively, and are in full parallelism with Eq. (3), whereas the eigenvectors of the macroscopic and differential matrices, i.e., $V_{\mathrm{M}}$ and $V_{\mathrm{m}}$, respectively, are the same. ${ }^{17}$ Assuming that $m_{\lambda}$ is a diagonal matrix with nonzero diagonal elements of $\lambda(\mathrm{m})$, the differential Mueller matrix can be obtained from an eigen analysis of $\mathrm{M}$ as follows:

$$
\mathrm{m}=V_{M} m_{\lambda} V_{M}^{-1}\left[\begin{array}{llll}
m_{11} & m_{12} & m_{13} & m_{14} \\
m_{21} & m_{22} & m_{23} & m_{24} \\
m_{31} & m_{32} & m_{33} & m_{34} \\
m_{41} & m_{42} & m_{43} & m_{44}
\end{array}\right]
$$

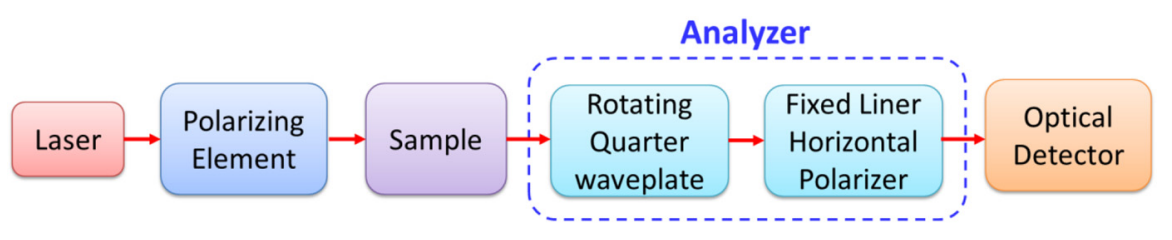

Fig. 1 Rotating quarter waveplate method for measuring Stokes parameters. 
In accordance with the differential Mueller matrix method, ${ }^{18}$ the Mueller matrix of a general anisotropic sample can be partitioned into 16 different elements, with each element describing a different aspect of the optical behavior. Let $\mathrm{M}_{\mathrm{LB}}$, $\mathrm{M}_{\mathrm{CB}}, \mathrm{M}_{\mathrm{LD}}$, and $\mathrm{M}_{\mathrm{CD}}$ be the macroscopic Mueller matrices describing the $\mathrm{LB}, \mathrm{CB}, \mathrm{LD}, \mathrm{CD}$, and Dep properties of the sample, respectively. The corresponding differential Mueller matrices, i.e., $\mathrm{m}_{\mathrm{LB}}, \mathrm{m}_{\mathrm{CB}}, \mathrm{m}_{\mathrm{LD}}, \mathrm{m}_{\mathrm{CD}}$ and $\mathrm{m}_{\Delta}$, can be found in Ref. 11. Thus, the differential Mueller matrix describing all of the $\mathrm{LB}, \mathrm{CB}, \mathrm{LD}, \mathrm{CD}$, and Dep properties of the anisotropic optical sample can be obtained by summing $\mathrm{m}_{\mathrm{LB}}, \mathrm{m}_{\mathrm{CB}}, \mathrm{m}_{\mathrm{LD}}$, $\mathrm{m}_{\mathrm{CD}}$, and $\mathrm{m}_{\Delta}$ to give ${ }^{10}$

$$
\begin{aligned}
& m_{\mathrm{BD} \Delta}=m_{\mathrm{BD}}+m_{\Delta} \\
&=\frac{1}{d}\left[\begin{array}{cc}
\ln \left[\left(1-R^{2}\right) \sqrt{\frac{1-D}{1+D}}\right] & -\ln \sqrt{\frac{1-D}{1+D}} \cos \left(2 \theta_{d}\right)+\kappa_{q}^{\prime} \\
-\ln \sqrt{\frac{1-D}{1+D}} \cos \left(2 \theta_{d}\right)-\kappa_{q}^{\prime} & \ln \left[\left(1-R^{2}\right) \sqrt{\frac{1-D}{1+D}}\right]-\kappa_{i q}^{\prime} \\
-\ln \sqrt{\frac{1-D}{1+D}} \sin \left(2 \theta_{d}\right)-\kappa_{u}^{\prime} & -2 \gamma+\eta_{v}^{\prime} \\
\ln \left(\frac{1+R}{1-R}\right)-\kappa_{v}^{\prime} & \beta \sin (2 \alpha)+\eta_{u}^{\prime}
\end{array}\right.
\end{aligned}
$$

where $\alpha$ is the eigenaxis orientation angle; $\beta$ is the linear phase retardance; $\gamma$ is the optical rotation angle; $\theta d$ is the orientation angle of the linear diattenuation; $D$ is the linear diattenuation; and $R$ is the circular diattenuation. Additionally, $d$ is the sample thickness and Dep is characterized by the differential parameters $\kappa_{i q, i u, i v}^{\prime}$, whereas the anomalous dichroism and anomalous Dep are characterized by the differential parameters $\kappa_{q, u, v}^{\prime}$ and $\eta_{q, u, v}^{\prime}$, respectively. From Eq. (5), $\alpha, \beta, \gamma, \theta_{d}, D$, and $R$ can be obtained, respectively, as follows: ${ }^{10}$

$\alpha=\frac{1}{2} \tan ^{-1}\left(\frac{m_{42}-m_{24}}{m_{34}-m_{43}}\right)$,

$\beta=\sqrt{\left(\frac{m_{42}-m_{24}}{2}\right)^{2}+\left(\frac{m_{34}-m_{43}}{2}\right)^{2}}$,

$\gamma=\left(\frac{m_{23}-m_{32}}{4}\right)$

$\theta_{\mathrm{d}}=\frac{1}{2} \tan ^{-1}\left(\frac{m_{13}+m_{31}}{m_{12}+m_{21}}\right)$

$D=\frac{1-e^{\sqrt{\left(m_{12}^{2}+m_{21}^{2}\right)+\left(m_{13}^{2}+m_{31}^{2}\right)}}}{1+e^{\sqrt{\left(m_{12}^{2}+m_{21}^{2}\right)+\left(m_{13}^{2}+m_{31}^{2}\right)}}}$,

and

$R=\frac{e^{\left(\frac{\left(m_{14}+m_{41}\right)}{2}\right)}-1}{e^{\left(\frac{\left(m_{14}+m_{41}\right)}{2}\right)}+1}$.

In addition, the differential Mueller matrix describing the Dep effect can be obtained as follows: ${ }^{10}$

$$
\left.\begin{array}{cc}
-\ln \sqrt{\frac{1-D}{1+D}} \sin \left(2 \theta_{d}\right)+\kappa_{u}^{\prime} & \ln \left(\frac{1+R}{1-R}\right)+\kappa_{v}^{\prime} \\
2 \gamma+\eta_{v}^{\prime} & -\beta \sin (2 \alpha)+\eta_{u}^{\prime} \\
\ln \left[\left(1-R^{2}\right) \sqrt{\frac{1-D}{1+D}}\right]-\kappa_{i u}^{\prime} & \beta \cos (2 \alpha)+\eta_{q}^{\prime} \\
-\beta \cos (2 \alpha)+\eta_{q}^{\prime} & \ln \left[\left(1-R^{2}\right) \sqrt{\frac{1-D}{1+D}}\right]-\kappa_{i v}^{\prime}
\end{array}\right],
$$

$$
\mathrm{m}_{\Delta}=\left[\begin{array}{cccc}
0 & \frac{\left(m_{21}-m_{12}\right)}{2} & \frac{\left(m_{31}-m_{13}\right)}{2} & \frac{\left(m_{41}-m_{14}\right)}{2} \\
\frac{\left(m_{21}-m_{12}\right)}{2} & \left(m_{22}-m_{11}\right) & \frac{\left(m_{23}+m_{32}\right)}{2} & \frac{\left(m_{24}-m_{42}\right)}{2} \\
\frac{\left(m_{31}-m_{13}\right)}{2} & \frac{\left(m_{23}+m_{32}\right)}{2} & \left(m_{33}-m_{11}\right) & \frac{\left(m_{34}+m_{43}\right)}{2} \\
\frac{\left(m_{41}-m_{14}\right)}{2} & \frac{\left(m_{24}-m_{42}\right)}{2} & \frac{\left(m_{34}+m_{43}\right)}{2} & \left(m_{44}-m_{11}\right)
\end{array}\right] .
$$

Performing inverse differentiation, ${ }^{15}$ the macroscopic matrix describing the Dep effect is obtained as follows:

$\mathrm{M}_{\Delta}=\left[\begin{array}{cccc}1 & K_{12} & K_{13} & K_{14} \\ -K_{12} & K_{22} & K_{23} & K_{24} \\ -K_{13} & K_{23} & K_{33} & K_{34} \\ -K_{14} & K_{24} & K_{34} & K_{44}\end{array}\right]$,

where $K_{22}$ and $K_{33}$ are the two-dimensional degrees of linear Dep and $K_{44}$ is the degree of circular Dep. In general, the degree of Dep is quantified by the Dep index $\Delta$, which has a value of 0 for a nondepolarizing sample and 1 for an ideal depolarizer. More specifically, the Dep index can be obtained as follows: $:^{19,20}$

$$
\Delta=1-\sqrt{\frac{K_{22}^{2}+K_{33}^{2}+K_{44}^{2}}{3}}, \quad 0 \leq \Delta \leq 1 .
$$

Moreover, the so-called effective LB parameters of an anisotropic sample can be extracted from Eq. (15) and using the LB-only model as follows:

$$
\begin{aligned}
& m_{l b}=\left|\begin{array}{cccc}
0 & 0 & 0 & 0 \\
0 & 0 & 0 & -\beta \sin (2 \alpha) \\
0 & 0 & 0 & \beta \cos (2 \alpha) \\
0 & \beta \sin (2 \alpha) & -\beta \cos (2 \alpha) & 0
\end{array}\right|, \\
& \alpha=\frac{1}{2} \tan ^{-1}\left(\frac{m_{24}}{m_{34}}\right),
\end{aligned}
$$

and 
$\beta=\frac{m_{34}-m_{43}}{2 \cos (2 \alpha)}$.

\subsection{Experimental Setup}

The feasibility of the proposed all-parameter decoupled model was investigated using chicken breast muscle tissue. According to the data of Food and Agriculture Organization of the united nationals, ${ }^{18}$ meat is composed of water, fat, protein, minerals, and a small proportion of carbohydrate. The fat of fresh chicken meat, pork (lean), beef (lean), beef carcass and pork carcass is $0.9 \%, 1.2 \%, 1.8 \%, 28 \%$, and $47 \%$, respectively. The chicken breast tissue was deliberately chosen as the sample material since it has a clear texture direction and compared to porcine or bovine muscle tissue has less adipose tissue.

Figure 2 presents a schematic illustration of the experimental full-field Mueller matrix ellipsometry setup. As shown, the main items of equipment include a laser illumination source (JDS Uniphase He-Ne laser, 1144P) with an output power of $15 \mathrm{~mW}$ and a working wavelength of $632.8 \mathrm{~nm}$, a CCD camera (1500MT1-GE, DVC, high QE CCD: $>62 \%$ at $500 \mathrm{~nm}$ ), a step motor, a digital force gage, and a computer. In addition, an input polarizing system comprises a beam expander (BE052-A, Thorlabs, $0.5 \times$ to $2 \times$ ), a neutral density filter (NDC-100, Qnset), a polarizer (SPF-30C-32, Qnset, 400 to $700 \mathrm{~nm}$ ), and a quarter waveplate (WPQ10M-633, Thorlabs, $\lambda / 4$ at $633 \mathrm{~nm}$ ). An analyzer module consists of a rotating quarter waveplate (WPQ10M-633, Thorlabs, $\lambda / 8$ at $633 \mathrm{~nm}$ ) and a fixed polarizer (SPF-30C-32, Qnset, 400 to $700 \mathrm{~nm}$ ).

In performing the experimental tests, the tissue sample was subjected to tensile loads ranging from 0 to $1 \mathrm{~N}$ in increments of $0.2 \mathrm{~N}$. For each value of the applied load and each input polarized light, the eigenaxis orientation of the polarizer in the analyzer was set to the horizontal direction while the quarter waveplate was rotated to $0,22.5,45,67.5,90,112.5,135$, and $157.5 \mathrm{deg}$, respectively. The resulting CCD images were processed using the computer to obtain the Stokes vectors required to calculate the corresponding $\mathrm{LB}, \mathrm{CB}, \mathrm{LD}, \mathrm{CD}$,

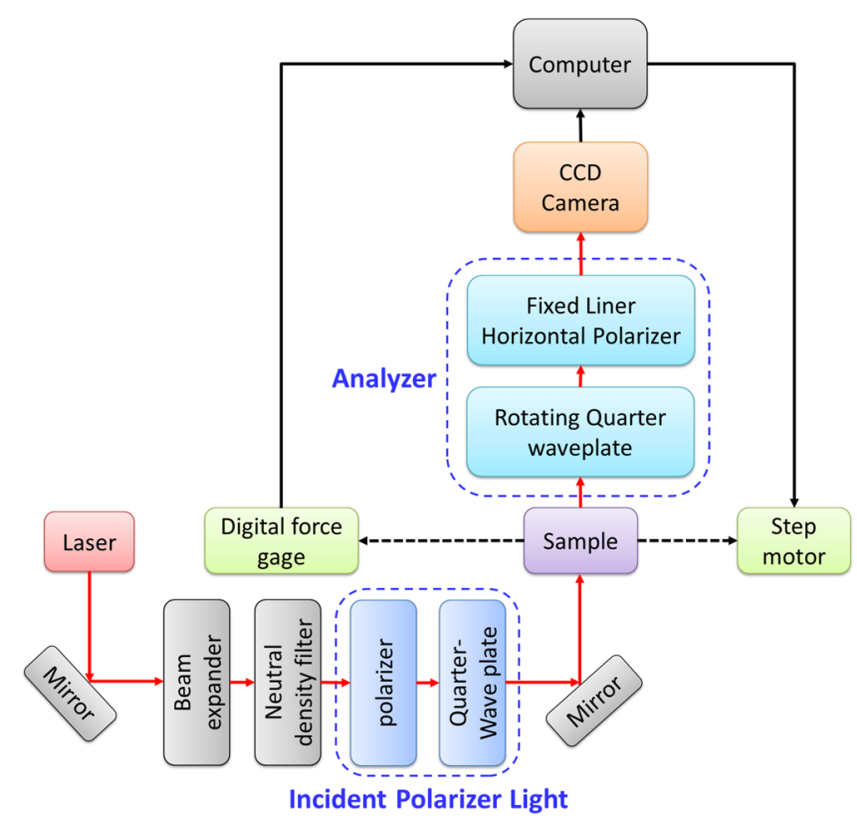

Fig. 2 Experimental setup of full-field Mueller matrix ellipsometer. and Dep properties of the sample using Eqs. (6)-(14). For comparison purposes, the LB properties of the sample were also determined using the LB-only model [i.e., Eqs. (16) and (17)].

The experimental setup is illustrated in Fig. 2 in performing the stretching tests with one sample applied load between 0 and $1 \mathrm{~N}$. Each sample had a size of $\sim 40 \mathrm{~mm} \times 20 \mathrm{~mm}$ (length $\times$ width) and the spot size of the incident light $(\mathrm{He}-$ $\mathrm{Ne}$ laser) $<2 \mathrm{~mm}$. It is noted that the fixture with glue on the edge of the soft sample is designed in order to ensure the uniform tension on a sample before the fracture forming on the edge.

Moreover, the thickness was carefully controlled to $2 \mathrm{~mm}$ since a thicker sample was found to block the incident laser beam while a thinner sample was found to break under the effects of the applied load. Furthermore, the tests were performed using fresh chicken tissue since as the tissue protein decomposes under putrefaction and the sample property changes. Furthermore, in every case, the sample was oriented in such a way that the stretching force acted in a perpendicular direction to that of the muscle fiber. It is noted that the muscle fiber would be compressed and the arrangement of myofibril be extended when the stretching force acted in a perpendicular direction to that of the muscle fiber, and thus make the LB increased.

\section{Results and Discussion}

\subsection{Performance of Different Models on Linear Birefringence Extraction}

The LB-only model uses a simple photoelasticity model to extract the LB parameters ( $\alpha$ and $\beta$ ) of anisotropic samples. In other words, the effects of the other optical properties of the sample (i.e., CB, LD, CD, and Dep) on the $\mathrm{LB}$ response are ignored. To evaluate the resulting error in the extraction results, a series of simulations was performed in which the LB results obtained using the LB-only model were compared with those obtained using the all-parameter decoupled model for four hypothetical anisotropic samples with hybrid $(\mathrm{LB}+\mathrm{Dep}),(\mathrm{LB}+\mathrm{CB}),(\mathrm{LB}+\mathrm{LD})$, and $(\mathrm{LB}+\mathrm{CD})$ properties, respectively. The corresponding results are shown in Figs. 3(a)3(h) below.

\subsubsection{Effect of depolarization property}

The differential Mueller matrix of a hybrid sample with mixed LB and Dep properties was simulated. Figures 3(a) and 3(b) compare the results obtained by the LB-only model and the all-parameter decoupled model for the LB property of a hybrid sample with various degrees of Dep (i.e., various values of the Dep index). It is seen that for both models, the extracted LB is identical to the input LB. In other words, the extraction performance of the LB-only model is robust toward the effects of Dep. This finding confirms that when using the LB-only model to evaluate the LB property of a sample with the traditional photoelastic coating, the surface roughness-induced Dep effect can be ignored. ${ }^{21}$

\subsubsection{Effect of circular birefringence property}

The differential Mueller matrix of a hybrid material with mixed LB and CB properties was simulated. Figures 3(c) and 3(d) 


\section{LB-only model}

(a)

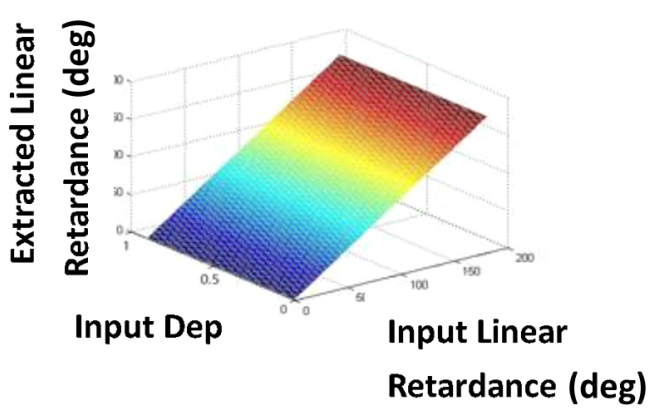

(c)

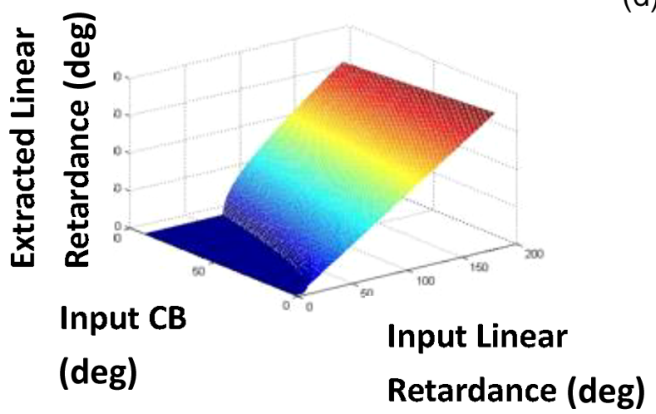

(d)

\section{all-parameter decoupled model}

(b)
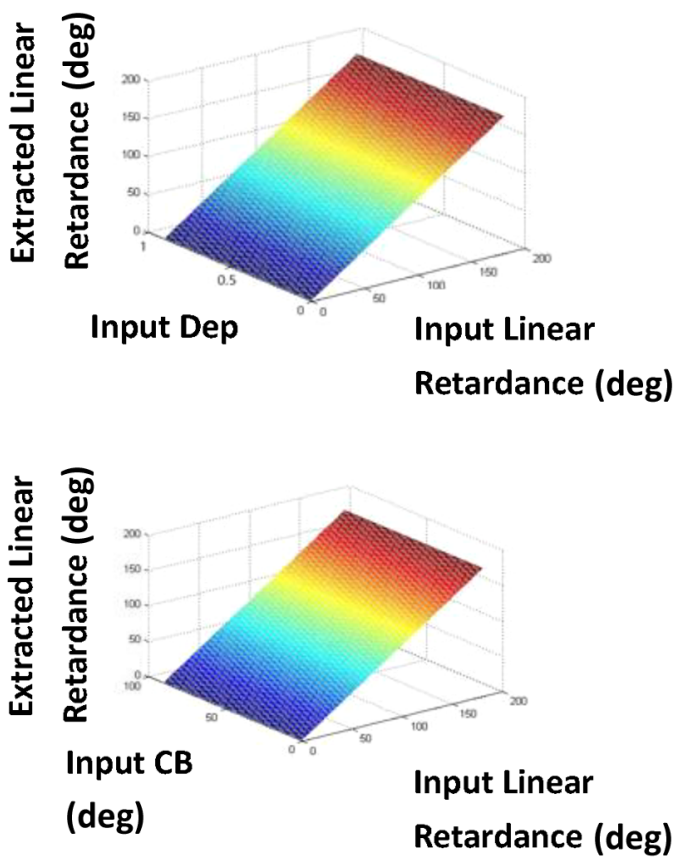

(e)

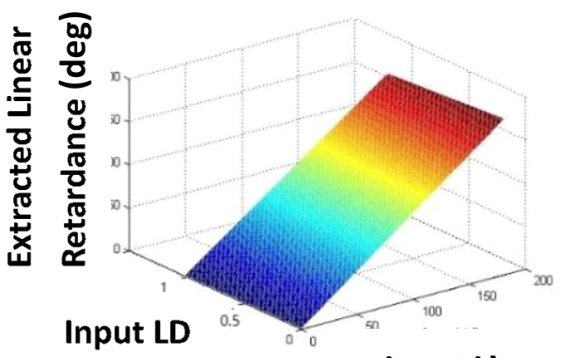

Input Linear

Retardance (deg)

(f)

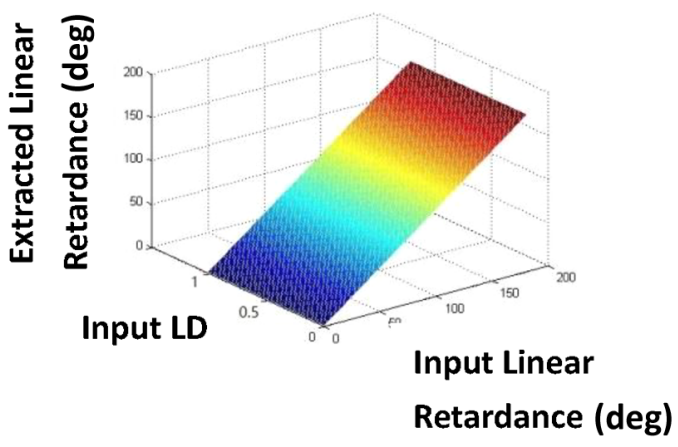

(g)

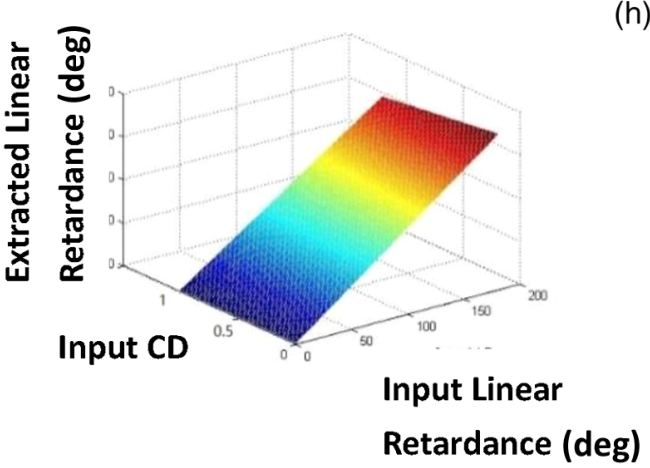

(h)

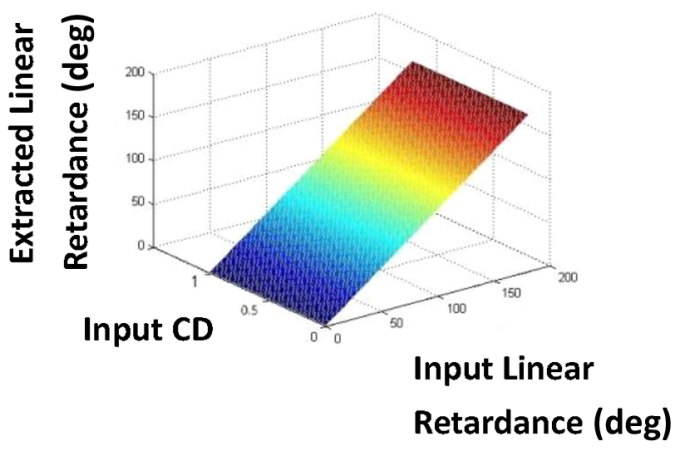

Fig. 3 (a) Linear retardance extraction obtained using LB-only model for hypothetical optical samples with (LB + Dep), (b) Linear retardance extraction obtained using all-parameter decoupled model for hypothetical optical samples with (LB + Dep), (c) Linear retardance extraction obtained using LB-only model for hypothetical optical samples with $(\mathrm{LB}+\mathrm{CB})$, (d) Linear retardance extraction obtained using all-parameter decoupled model for hypothetical optical samples with $(L B+C B)$, (e) Linear retardance extraction obtained using LB-only model for hypothetical optical samples with (LB + LD), (f) Linear retardance extraction obtained using all-parameter decoupled model for hypothetical optical samples with (LB + LD), (g) Linear retardance extraction obtained using LB-only model for hypothetical optical samples with $(L B+C D)$, (h) Linear retardance extraction obtained using all-parameter decoupled model for hypothetical optical samples with $(\mathrm{LB}+\mathrm{CD}$ ). (The range of input linear retardance, input $\mathrm{CB}$ and extracted linear retardance is 0 to $200 \mathrm{deg}$. The range of input Dep, input LD, and input CD is 0 to 1.) 
show the LB extraction results obtained by the two models for optical samples with various degrees of CB. For the all-parameter decoupled model, the extracted LB is identical to the input LB. However, it is seen that the LB-only model underestimates the LB property of the hybrid sample. Moreover, the extraction error increases with an increasing CB.

\subsubsection{Effect of linear dichroism property}

The differential Mueller matrix of a hybrid material with mixed LB and LD properties was simulated. Figures 3(e) and 3(f) show the LB extraction results obtained using the two models. For the all-parameter decoupled model, the extracted LB values are in good agreement with the input values. However, for the LB-only model, a close inspection shows that the extracted values of the LB are slightly lower than those of the input LB. Moreover, the extraction error increases with an increasing LD.

\subsubsection{Effect of circular dichroism property}

The differential Mueller matrix of a hybrid material with mixed LB and CD properties was simulated. Figures $3(\mathrm{~g})$ and $3(\mathrm{~h})$ compare the LB extraction results obtained by the two models for optical samples with various degrees of $C D$. The $L B$ values extracted using the all-parameter decoupled model are almost identical to the input values for all values of the $C D$. However, for the LB-only model, the extracted LB values are once again slightly lower than the input values. Again, the extraction error increases with an increasing CD.

Overall, the simulation results presented in Fig. 3 show that for hybrid anisotropic samples, the all-parameter decoupled model enables the LB properties of the sample to be reliably determined irrespective of the other optical properties of the sample (i.e., Dep, CB, LD, and CD). By contrast, while the LB-only model provides a good extraction performance for samples with combined Dep and LB properties, the extraction results are less satisfactory for hybrid samples with $\mathrm{CB}, \mathrm{LD}$, and $\mathrm{CD}$ properties. In particular, the performance of the LB-only model is seriously degraded for samples with high CB.

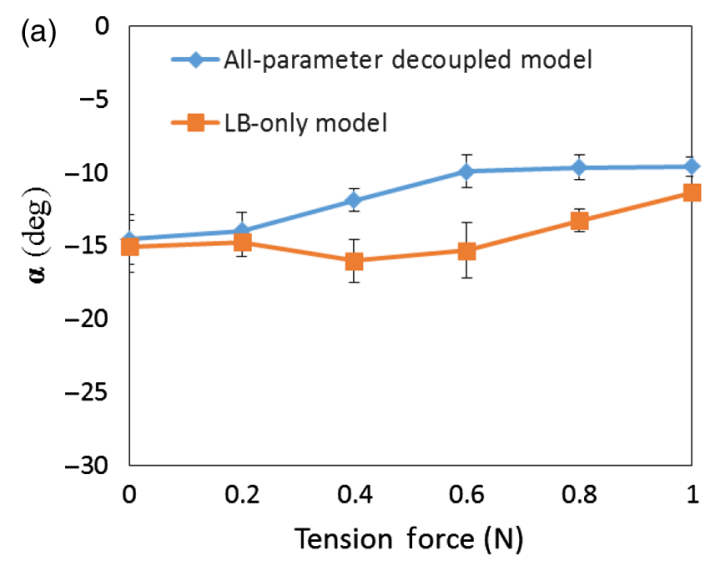

\subsection{Anisotropic Optical Properties of Normal Chicken Breast Tissue}

All the experimental data are used, the photos taken by CCD camera and the 2-D images are saved as a Tagged Image File Format files to transform as the matrix for the subsequent calculation. The photos are $1392 \times 1040$ pixels and it can be transformed to a $1392 \times 1040$ data matrix. The experimental data are randomly captured in this data matrix in order to estimate the sample property.

It is noted that before the chicken tissue sample is conducted in experiments, the optical system is characterized in its performance by testing the standard sample (quarter waveplate). As a result, the standard deviations in extracting $\alpha, \beta, \gamma, \theta_{d}, D, R$, and $\Delta$ of a quarter waveplate are $0.091 \mathrm{deg}, 0.493 \mathrm{deg}, 0.205 \mathrm{deg}$, $0.180 \mathrm{deg}, 0.040,0.003$, and 0.078 , respectively. It is noted that all the orientations of the eigenaxis of the waveplate and polarizer in an optical system are known and examined. Therefore, $\alpha$, $\beta, \gamma, \theta_{d}, D, R$, and $\Delta$ of the tested sample can be precisely defined and extracted.

Figure 4 shows the results obtained from the LB-only model and all-parameter decoupled model, respectively, for the eigenaxis orientation angle, $\alpha$, and linear phase retardation, $\beta$, of the chicken tissue samples stretched under the considered tensile loads. It is observed that for both models, the eigenaxis orientation angle remains approximately constant as the tensile load increases, but the phase retardance increases. In other words, the results are consistent with those reported in Ref. 6 using a PS-OCT technique. Nonetheless, the good general agreement between the results obtained using the two different models confirms that the LB-only model is robust toward the effects of scattering within the sample [see Fig. 2(a)]. The sensitivity is found around $21.42 \mathrm{deg} / \mathrm{N}$. For general anisotropic optical samples, the linear phase retardance $\beta$ is given by $\beta=k d\left(n_{\mathrm{e}}-n_{\mathrm{o}}\right)$, where $k$ is $2 \pi / \Lambda, d$ is the sample thickness, $n_{\mathrm{e}}$ is the extraordinary refractive index of the sample, $n_{\mathrm{o}}$ is the ordinary refractive index, and $\Lambda$ is the laser wavelength. In the present tests, the tensile force is applied perpendicularly to the fiber direction. Consequently, the sample density and ordinary refractive index reduce along the stretching axis. By contrast, the extraordinary refractive index increases, and hence, the linear phase retardance increases with an increasing tensile force. Moreover, the

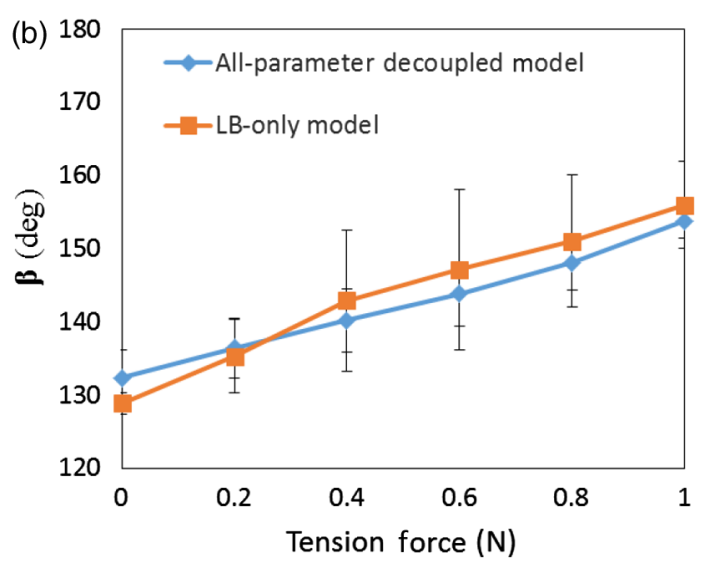

Fig. 4 LB properties of chicken muscle tissue under stretching as evaluated using LB-only model and all-parameter decoupled model. (a) Eigenaxis orientation angle, $\alpha$, obtained using LB-only model and all-parameter decoupled model. (b) Linear phase retardation, $\beta$, obtained using LB-only model and allparameter decoupled model. 

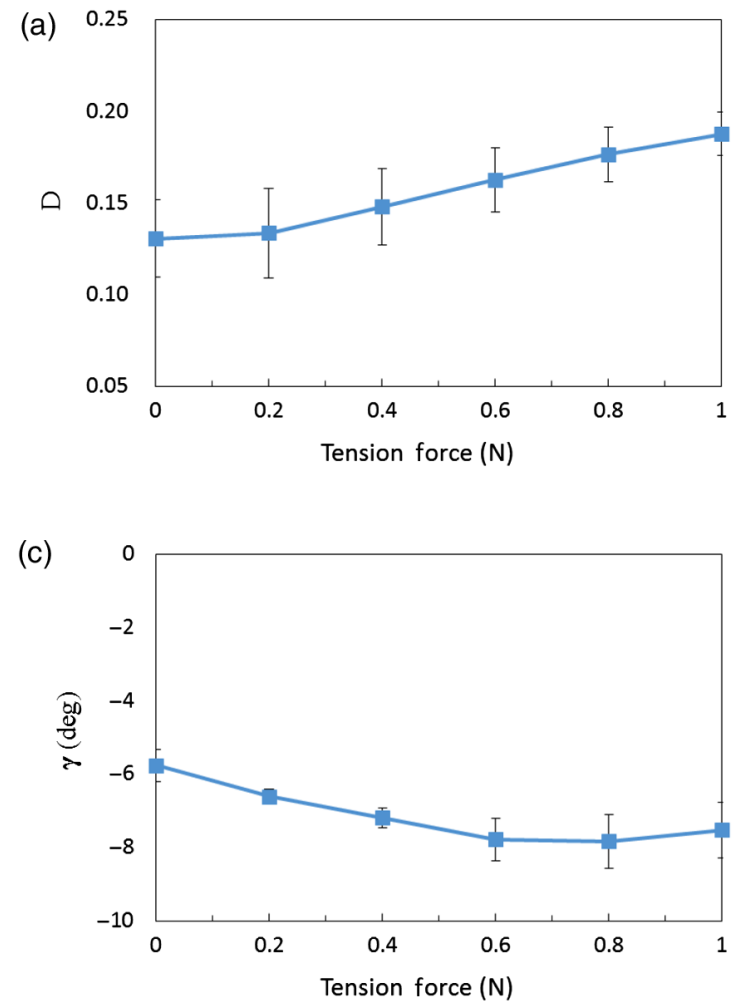
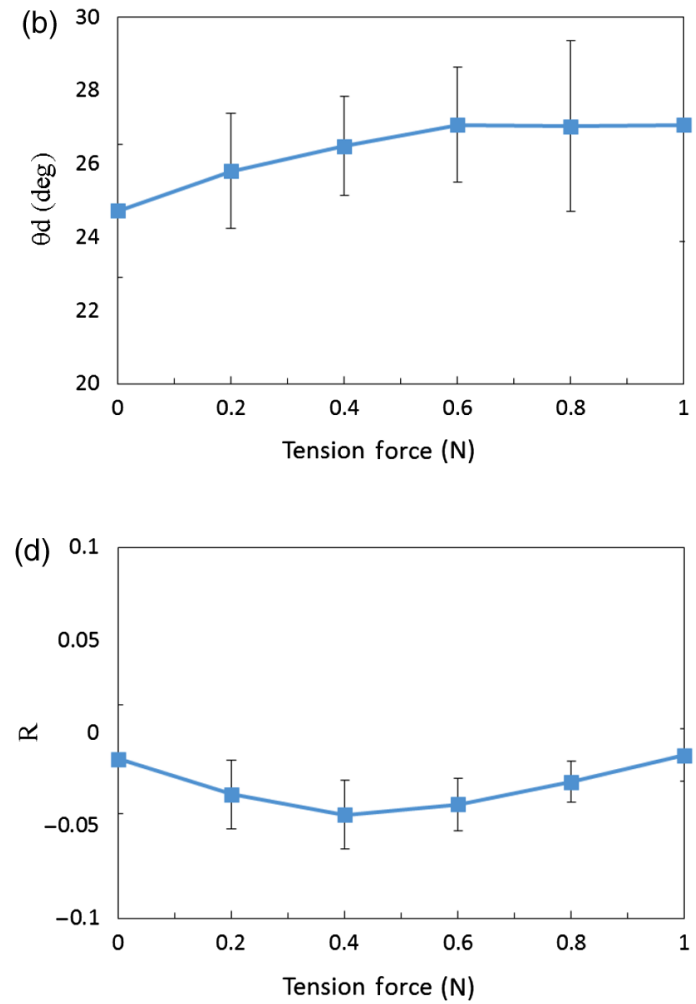

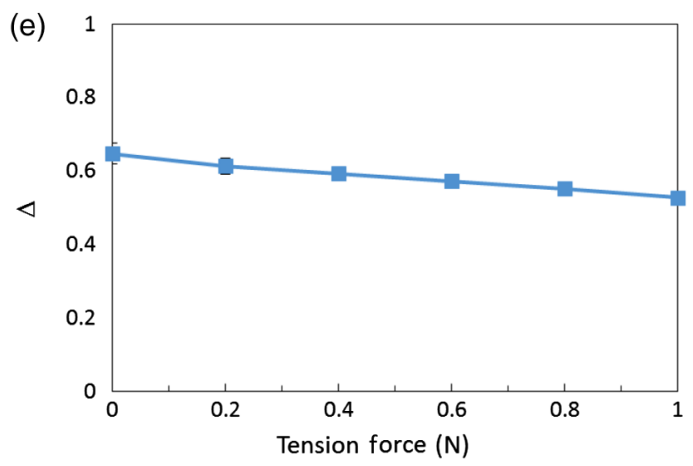

Fig. 5 Results obtained by all-parameter decoupled model for $D, \theta_{d}, \gamma, R$, and $\Delta$ properties of chicken muscle tissue under stretching. (a) Linear diattenuation, $D$, obtained by all-parameter decoupled model. (b) The orientation angle of the linear diattenuation, $\theta_{d}$, obtained by all-parameter decoupled model. (c) The optical rotation angle, $\gamma$, obtained by all-parameter decoupled model. (d) Circular diattenuation, $R$, obtained by all-parameter decoupled model. (e) Depolarization index, $\Delta$, obtained by all-parameter decoupled model.

extracted $\beta$ regarding to different loadings is calculated by data of 48 images (by six different input lights and eight rotation angles) and finally, it is averaged. It is found that the increments of $\beta(\sim 4 \mathrm{deg})$ regarding to the increment in $0.2 \mathrm{~N}$ tensile force is much larger than the standard deviation in 0.493 deg with a standard sample (quarter waveplate); thus, the extracted $\beta$ in Fig. 4 is obviously influenced by the tensile force. In addition, the error bars reflect that the data extracted by the all-parameter model are more stable in this case.

Figure 5 shows the results obtained from the all-parameter decoupled model for the variation of the $\mathrm{LD}, \mathrm{CB}, \mathrm{CD}$, and Dep properties of the chicken muscle tissue under the effects of an increasing tensile force. It can be seen that the linear diattenuation, $D$, increases slightly with an increasing force, while the diattenuation axis angle $\theta_{d}$ remains approximately constant.
It is found that the standard deviation of standard sample (systematic errors) in extracting $D$ is 0.040 , so the extracted $D$, varied from 0.15 to 0.19 , is reliable in its trend with applied force. However, the extracted $\theta_{d}$ is ranged $\sim 2 \mathrm{deg}$ and their standard deviations are more than $2 \mathrm{deg}$. Thus, the diattenuation axis angle $\theta_{d}$ remains approximately constant. In other words, the LD response of the muscle tissue under tension is similar to that of the $\mathrm{LB}$ response.

In Fig. 5, the extracted $\gamma$ is ranged from -5.76 to $-7.83 \mathrm{deg}$ and the standard deviation of standard sample (systematic errors) in extracting $\gamma$ is $0.205 \mathrm{deg}$; thus, the extracted $\gamma$ is reliable in its trend with applied force. However, the extracted $\gamma$ is ranged $\sim 2$ deg and their standard deviations are around $2 \mathrm{deg}$. As a result, the extracted $\gamma$ shows no observable trend as the tensile force increases. Additionally, the extracted $R$ is ranged 

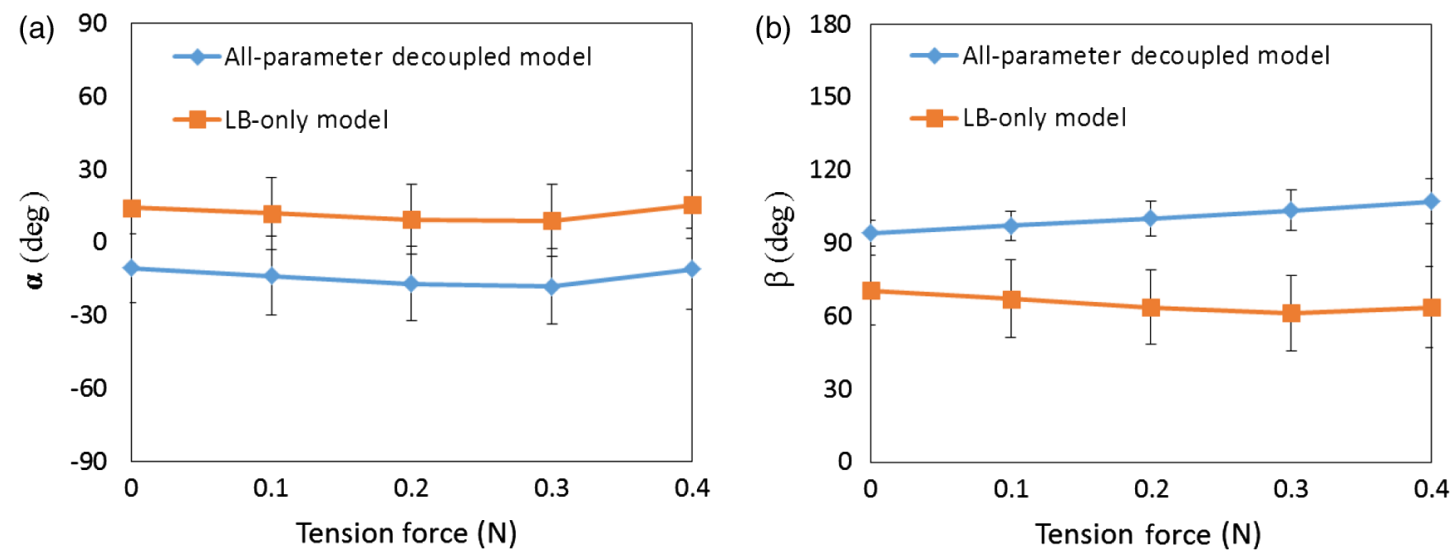

Fig. 6 Results obtained by LB-only model and all-parameter decoupled model for LB property of chicken muscle tissue with high CB. (a) Eigenaxis orientation angle, $\alpha$, obtained using LB-only model and all-parameter decoupled model. (b) Linear phase retardation, $\beta$, obtained using LB-only model and all-parameter decoupled model.

from -0.01 to -0.04 and the standard deviation of standard sample (systematic errors) is 0.003 , so the extracted $R$ is reliable in its trend with applied force. However, the extracted $R$ is ranged $\sim 0.03$ and their standard deviations are more than 0.03 . Thus, the extracted $R$ shows no observable trend as the tensile force increases. It is concluded that the extracted values of $\gamma$ and $R$ show no observable trend as the tensile force increases. The result is reasonable since chicken breast tissue is dominated by fibrous muscle structure and without dextrorotatory and levorotatory structures, such as glucose. These structures are unaffected by the application of a tensile force and thus, whereas the $\mathrm{CB}$ and $\mathrm{CD}$ properties are sensitive to phase change and attenuation of right-hand and left-hand circular polarization light, they remain unchanged as the tensile force increases.

The Dep index, $\Delta$, reduces with an increasing tensile force. The extracted $\Delta$ is ranged from 0.65 to 0.53 and the standard deviation of standard sample (systematic errors) is 0.078 ; therefore, the extracted $\Delta$ is reliable in its trend with applied force. The extracted $\Delta$ is ranged $\sim 0.12$ and their standard deviations are obviously $<0.12$. As a result, the extracted $\Delta$ shows the linear trend as the tensile force increases. It can be induced by thinner thickness and density to influence the less scattering while the tensile force is applied.

\subsection{Extracted Linear Birefringence Property of Chicken Breast with Additional Circular Birefringence Property}

Because the samples (chicken breast tissue) are naturally devoid of the $\mathrm{CB}$ property, adding glucose solution into the chicken breast tissue verifies and demonstrates the simulation in Figs. 3(c) and 3(d). It is found that a special sample with LB and $\mathrm{CB}$ properties cannot be analyzed properly if the LBonly model is applied. Chicken breast samples with high $\mathrm{CB}$ were prepared by immersing the breast tissue in a glucose solution with a concentration of $16009 \mathrm{mg} / \mathrm{dl}(0.9 \mathrm{M})$ for 5 days. As described above, for healthy chicken breast tissue, the LB property extracted by the LB-only model is in good agreement with that obtained using the all-parameter decoupled model for all considered values of the tensile force (see Fig. 4).

Figure 6 shows the results of chicken muscle tissue with high $\mathrm{CB}$ under the loading only from 0 to $0.4 \mathrm{~N}$. It is because the tested sample immersed in glucose solution becomes weaker for tensile tests. As shown in Fig. 6, for the chicken sample with $\mathrm{CB}$ property, the $\mathrm{LB}$ results extracted by the LB-only model are underestimated for all values of the tensile force. Furthermore, the values of $\alpha$ and $\beta$ extracted using the LBonly model exhibit a greater variance than those extracted using the all-parameter decoupled model. Interestingly, the trends of the extracted $\beta$ by two models are totally opposite. Also, it is found that the trend of the extracted $\beta$ by all-parameter decoupled model here is similar to the one in Fig. 4(b). The slope in Fig. 6 is found around $32.75 \mathrm{deg} / \mathrm{N}$ and it is higher than the one, $21.42 \mathrm{deg} / \mathrm{N}$, calculated in Fig. 4(b). It is noted that the normal muscle exhibits a clear anisotropic structure that chicken meat is composited of $75 \%$ water, $22.8 \%$ protein, $0.9 \%$ fat, and $1.2 \%$ ash. $^{18}$ When the sample is immersed into glucose solution, a hypertonic solution, the sample would be dehydrated and osmotic shrinkage. Thus, the glucose makes the muscle tissue dehydrated and thus enhances the fibrous texture more dense and arranged. In general, the experimental results are consistent with the simulation results presented in Figs. 3(c) and 3(d) and confirm that for samples with CB property, the allparameter decoupled model provides a more robust approach for extracting LB property.

\section{Conclusions}

The LB property of biological tissues under stretching provides a useful indication of potential changes in the physical function, metabolism, and pathology of the tissue, and are thus of significant interest in many disease diagnosis applications. For example, type 2 diabetes may translate into functional impairment in the elder people and this may reflect a link between the mechanical functions of muscle ${ }^{22,23}$ and the muscle birefringence (LB) is due to the well-arranged muscle structures. The LB property is traditionally extracted based on a simple photoelasticity model. However, the extraction process ignores the effects of the other optical properties of the anisotropic sample on the LB response, and hence, the accuracy of the extracted LB results cannot be guaranteed. Accordingly, the present study has proposed an LB extraction method based on a differential Mueller matrix model and fullfield ellipsometry, in which the LB property of the sample is decoupled from the $\mathrm{CB}, \mathrm{LD}, \mathrm{CD}$, and Dep properties. 
Simulations have been performed to compare the performance of the traditional "LB-only" model and "all-parameter" decoupled model in extracting the LB property of four hypothetical anisotropic optical samples with hybrid (LB+Dep), $(\mathrm{LB}+\mathrm{CB}),(\mathrm{LB}+\mathrm{LD})$, and $(\mathrm{LB}+\mathrm{CD})$ properties. The results have shown that while the all-parameter decoupled model accurately extracts the LB property of all four samples, the accuracy and repeatability of the LB-only model are severely degraded for a sample with $\mathrm{CB}$ property. Notably, however, the LB-only model is robust toward the effects of scatteringinduced Dep.

The practical feasibility of the proposed method has been demonstrated experimentally using chicken breast muscle tissue samples subjected to tensile loads ranging from 0 to $1.0 \mathrm{~N}$. It has been shown that the linear phase retardance $(\beta)$, linear diattenuation $(D)$, and Dep index $(\Delta)$ of healthy chicken breast tissue increase with an increasing tensile load. However, the rotational angle $(\gamma)$ and circular diattenuation $(R)$ remain approximately constant as the tensile load is increased. For a chicken breast sample with high $\mathrm{CB}$, the all-parameter decoupled model retains a good LB extraction performance. However, the LB results extracted using the LB-only model not only exhibit a greater variance than those extracted using the all-parameter decoupled model but are also underestimated.

In summary, the results presented in this study show that the traditional LB-only model provides a reliable LB extraction performance for optical samples with strong LB or scatteringinduced Dep. However, the proposed all-parameter decoupled model not only enables all of the optical properties of an anisotropic optical sample to be extracted but also provides a more accurate and reliable measurement of the LB property for samples with high $\mathrm{CB}$.

\section{Disclosures}

No conflicts of interest, financial or otherwise, are declared by the authors.

\section{Acknowledgments}

The authors gratefully acknowledge the financial support provided to this study by the Ministry of Science and Technology (MOST) in Taiwan under Grant Nos. MOST-104-2221-E-006125-MY2 and MOST-104-2221-E-006-114-MY2. The partial support of this study by the Headquarters of University Advancement at National Cheng Kung University (NCKU) under the sponsorship of the Ministry of Education, Taiwan, is also much appreciated.

\section{References}

1. A. S. Szczesniak, M. A. Brandt, and H. H. Friedman, "Development of standard rating scales for mechanical parameters of texture and correlation between the objective and the sensory methods of texture evaluation," J. Food Sci. 28, 397-403 (1963).

2. D. W. Stanley et al., "Predicting meat tenderness from muscle tensile properties," J. Texture Stud. 3, 51-68 (1972).

3. W. F. Cheong, S. A. Prahl, and A. J. Welch, "A review of the optical properties of biological tissues," IEEE J. Quantum Electron. 26, 21662185 (1990).

4. R. Liao et al., "Rotating linear polarization imaging technique for anisotropic tissues," J. Biomed. Opt. 15, 036014 (2010).

5. M. F. G. Wood et al., "Polarization birefringence measurements for characterizing the myocardium, including healthy, infarcted, and stem-cell-regenerated tissues," J. Biomed. Opt. 15, 047009 (2010).
6. D. Chen et al., "Characterization of muscle stretching and damage using polarization-sensitive optical coherence tomography (PS-OCT)," Proc. SPIE 8553, 85533C (2012).

7. T. Dobashi et al., "An analysis of anisotropic gel forming process of chitosan," Carbohydr. Polym. 84, 709-712 (2011).

8. L. Csoka et al., "Polarization optical-histochemical characterization and supramolecular structure of carbohydrate fibrils," Acta Histochem. 115, 22-31 (2013).

9. Q.-H. Phan, Y.-L. Lo, and C.-L. Huang, "Surface plasmon resonance prism coupler for enhanced circular dichroism sensing," Opt. Express 24(12), 12812-12824 (2016).

10. C.-C. Liao and Y.-L. Lo, "Extraction of anisotropic parameters of turbid media using hybrid model comprising differential- and decompositionbased Mueller matrices," Opt. Express 21, 16831-16853 (2013).

11. T.-T.-H. Pham and Y.-L. Lo, "Extraction of effective parameters of anisotropic optical materials using a decoupled analytical method," J. Biomed. Opt. 17, 025006 (2012).

12. J. W. Dally and W. F. Rile, Experimental Stress Analysis, 4th ed., College House Enterprises LLC, TN (2005).

13. B. D. Cameron et al., "Measurement and calculation of the two-dimensional backscattering Mueller matrix of a turbid medium," Opt. Lett. 23, 485-487 (1998).

14. B. Schaefer et al., "Measuring the Stokes polarization parameters," Am. J. Phys. 75, 163-168 (2007).

15. R. M. A. Azzam, "Propagation of partially polarized light through anisotropic media with or without depolarization: a differential $4 \times 4$ matrix calculus," J. Opt. Soc. Am. 68, 1756-1767 (1978).

16. R. Ossikovski, "Differential matrix formalism for depolarizing anisotropic media," Opt. Lett. 36, 2330-2332 (2011).

17. N. Ghosh, M. F. G. Wood, and I. A. Vitkin, "Polarimetry in turbid, birefringent, optically active media: a Monte Carlo study of Mueller matrix decomposition in the backscattering geometry," J. Appl. Phys. 105, 102023 (2009).

18. Meat, Fat and Other Edible Carcass Parts, Meat Processing Technology, Food and Agriculture Organization of the United Nationals, Bangkok (2007).

19. R. A. Chipman, "Depolarization index and the average degree of polarization," Appl. Opt. 44, 2490-2495 (2005).

20. B. J. DeBoo, J. M. Sasian, and R. A. Chipman, "Depolarization of diffusely reflecting man-made objects," Appl. Opt. 44, 5434-5445 (2005).

21. A. S. Redner, "Photoelastic coatings," Exp. Mech. 20, 403-408 (1980).

22. A. A. Sayer et al., "Type 2 diabetes, muscle strength, and impaired physical function," Diabetes Care 28(10), 2541-2542 (2005).

23. S. W. Park et al., "Decreased muscle strength and quality in older adults with type 2 diabetes: the health, aging, and body composition study," Diabetes 55(6), 1813-1818 (2006).

Hao-Wei Chen received his MS degree from the Department of Mechanical Engineering, National Cheng Kung University, Taiwan, China, in 2016.

Chih-Ling Huang received her BS, MS, and PhD degrees from the Department of Material Science and Engineering, National Cheng Kung University, Taiwan, China, in 2003, 2005, and 2010, respectively. After graduation, she has been a postdoctoral researcher in Department of Mechanical Engineering, National Cheng Kung University. Since 2016, she has been a member of the Center for Fundamental Science, Kaohsiung Medical University, where she is now an assistant professor.

Yu-Lung Lo received his $\mathrm{PhD}$ degree in mechanical engineering, University of Maryland, College Park, United States, in 1995. He has been a faculty of the Mechanical Engineering Department, NCKU, since 1996, where he is now a department head and distinguished professor. He was invited to be an invited speaker, keynote speaker, and plenary speaker in the international conferences. One of his articles is included in spotlight on optics by OSA in 2015.

You-Ren Chang received his BS degree from the Department of Mechanical Engineering, National Chung Hsing University, Taiwan in 2015. 